\title{
On One-Step Method of Euler-Maruyama Type for Solution of Stochastic Differential Equations Using Varying Stepsizes
}

\author{
Sunday Jacob Kayode1, Akeem Adebayo Ganiyu², Adegoke Sule Ajiboye ${ }^{3}$ \\ ${ }^{1}$ Department of Mathematical Sciences, Federal University of Technology, Akure, Nigeria \\ ${ }^{2}$ Mathematics Department, Adeyemi College of Education, Ondo, Nigeria \\ ${ }^{3}$ Department of Statistics, Federal University of Technology, Akure, Nigeria \\ Email: cosjkay57@yahoo.com, ganiyuiwajowa@gmail.com, asajib11@gmail.com
}

Received 9 January 2016; accepted 24 January 2016; published 29 January 2016

Copyright (C) 2016 by authors and OALib.

This work is licensed under the Creative Commons Attribution International License (CC BY). http://creativecommons.org/licenses/by/4.0/

(c) (i) Open Access

\begin{abstract}
In this work, a one-step method of Euler-Maruyama (EMM) type has been developed for the solution of general first order stochastic differential equations (SDEs) using Itô integral equation as basis tool. The effect of varying stepsizes on the numerical solution is also examined for the SDEs. Two problems of first order SDEs are solved. Absolute errors for the problems are obtained from which the mean absolute errors (MAEs) are calculated. Comparison of variation in stepsizes is achieved using the MAEs. The results show that the MAEs decrease as the stepsize decreases. The strong orders of convergence and the residuals for the problems are respectively obtained using Least Square Fit. This work produces numerical values for the solution to the problems at discritised points in a given interval which differ from the existing methods of EMM type where results are obtained by simulation.
\end{abstract}

\section{Keywords}

One-Step Method, Itô Integral, Stochastic State Model, Gaussian White Noise, Wiener Process, Wiener Increment

Subject Areas: Mathematical Analysis, Numerical Mathematics

\section{Introduction}

Modeling of physical systems by ordinary differential equations (ODEs) ignores stochastic effects. Addition of random elements into the differential equations leads to what is called stochastic differential equations (SDEs), and the term stochastic is called noise [1]. Many researchers have worked extensively on a first and higher order 
ordinary differential equations of the form

$$
X^{(n)}=f\left(t, X, X^{\prime}, \cdots, X^{n-1}\right), X^{(r)}\left(t_{0}\right)=X_{0}^{(r)}, t \in T, r=0,1, \cdots,
$$

where $f:[0, T] \times \mathbb{R}^{n} \rightarrow \mathbb{R}^{n}$. These researchers include [2]-[19].

Equation (1) is a deterministic state model which can be turned to a stochastic state model by including noise term. For one dimensional noise term, consider a general first order stochastic differential Equation (SDE) of the form

$$
X^{\prime}=f(t, X(t)) \mathrm{d} t+g(t, X(t)) \cdot \gamma, X\left(t_{0}\right)=X_{0}
$$

where $f:[0, T] \times \mathbb{R}^{n} \rightarrow \mathbb{R}^{n}$ and $g:[0, T] \times \mathbb{R}^{n} \rightarrow \mathbb{R}^{n \times m}$ drift function and diffusion function respectively. The noise $(\gamma)$ in Equation (2) is generally called Gaussian white noise. It is expressed as $\gamma=\frac{\mathrm{d} W(t)}{\mathrm{d} t}$, where $W(t)$ is the Wiener process.

Equation (2) can be written as

$$
\mathrm{d} X=f(t, X(t)) \mathrm{d} t+g(t, X(t)) \cdot \mathrm{d} W(t), X\left(t_{0}\right)=X_{0}
$$

Integrating (3) from 0 to $t$, we have Itô integral equation

$$
X(t)=X_{0}+\int_{0}^{t} f(s, X(s)) \mathrm{d} s+\int_{0}^{t} g(s, X(s)) \mathrm{d} W s
$$

The first integral at the right hand side of Equation (4) is called Riemman integral while the second integral is called Itô or stochastic integral. Many researchers have also worked on SDEs of the form (3). Amongst these are [20]-[29].

The aim of this paper is to develop numerical method for solution of first order stochastic differential Equation (3). Our objectives are to develop one-step Euler-Maruyama method (EMM) for solution of SDE (3) and apply it to solve two problems in the form of first order SDEs. Absolute errors (AEs) will be determined at various point in the interval $[0, T]$, where $T=1$. The AEs will be determined by finding the absolute value of the difference between the exact solution and numerical solution of Equation (3) for various values of $t \in[0, T]$ using one-step Euler-Maruyama method. These values will allow us to obtain mean absolute error (MAE).

The strong order of convergence (SOC) of the method will be determined from the results of the mean absolute errors obtained. [25] considered the effect of using single stepsize for solution of SDE (3). The first section of this paper introduces the content of the paper. The second section discusses the research methodology. Under this section, Itô integral form of stochastic differential Equation (3) stated in Equation (4) is used as the basis for the derivation of one-step Euler-Maruyama method. The method is implemented with two problems in the form of SDE in Equation (3). In section three, the effect of varying the stepsizes in determining the solution of SDE in Equation (3) is considered. The stepsizes used are $2^{-4}, 2^{-5}, 2^{-6}, 2^{-7}, 2^{-8}, 2^{-9}$. Numerical solution and absolute error are then obtained using each of the stepsize. Using ten values of $t \in[0,1]$, mean absolute errors are determined for each of the stepsizes. The SOC is calculated for each problem using the least square fit in [26]. MATLAB program is used as a supporting tool.

\section{Research Methodology}

There are many methods for determining the solution of SDE (3), they include, Euler-Maruyama method, Euler-Maruyama method, Runge-Kutta method, Heun method and so on. For more information about the list of methods or schemes for solution of first order SDEs (see [30]). To determine the solution of SDE (3), one-step method of Euler-Maruyama type will be used. Euler-Maruyama method was used by [26] for considering an autonomous system of stochastic differential equations. Here, we shall consider the derivation of the method using Itô integral Equation (4) obtained from a general form of the SDE stated in Equation (3).

\subsection{Derivation of One Step Euler-Maruyama Method}

One-step Euler-Maruyama method will be derived by setting $t=\tau_{j+r}, r=0,1$ in the integral

$$
X(t)=X_{0}+\int_{0}^{t} f(s, X(s)) \mathrm{d} s+\int_{o}^{t} g(s, X(s)) \mathrm{d} W(s)
$$


This gives

$$
\begin{gathered}
X\left(\tau_{j}\right)=X_{0}+\int_{0}^{\tau_{j}} f(s, X(s)) \mathrm{d} s+\int_{0}^{\tau_{j}} g(s, X(s)) \mathrm{d} W(s) \\
X\left(\tau_{j+1}\right)=X_{0}+\int_{0}^{\tau_{j+1}} f(s, X(s)) \mathrm{d} s+\int_{0}^{\tau_{j+1}} g(s, X(s)) \mathrm{d} W(s) \\
(6)-(5) \Rightarrow X\left(\tau_{j+1}\right)-X\left(\tau_{j}\right)=\int_{\tau_{j}}^{\tau_{j+1}} f(s, X(s)) \mathrm{d} s+\int_{\tau_{j}}^{\tau_{j+1}} g(s, X(s)) \mathrm{d} W(s) \\
\Rightarrow X\left(\tau_{j+1}\right)=X\left(\tau_{j}\right)+\int_{\tau_{j}}^{\tau_{j+1}} f(s, X(s)) \mathrm{d} s+\int_{\tau_{j}}^{\tau_{j+1}} g(s, X(s)) \mathrm{d} W(s)
\end{gathered}
$$

Using a conventional deterministic quadrature, the two integral terms at the right hand side can be approximated as follows:

$$
\begin{aligned}
& \int_{\tau_{j}}^{\tau_{j+1}} f(s, X(s)) \mathrm{d} s \approx \int_{\tau_{j}}^{\tau_{j+1}} f\left(\tau_{j}, X\left(\tau_{j}\right)\right) \mathrm{d} s=\int_{\tau_{j}}^{\tau_{j+1}} f\left(\tau_{j}, X\left(\tau_{j}\right)\right) \mathrm{d} s \\
&=f\left(\tau_{j}, X\left(\tau_{j}\right)\right) \int_{\tau_{j}}^{\tau_{j+1}} \mathrm{~d} s \\
& \int_{\tau_{j}}^{\tau_{j+1}} g(s, X(s)) \mathrm{d} W(s) \approx g\left(\tau_{j}, X\left(\tau_{j}\right)\right)\left(W\left(\tau_{j+1}\right)-W\left(\tau_{j}\right)\right)
\end{aligned}
$$

Substituting (8) and (9) in (7), we have

$$
X\left(\tau_{j+1}\right)=X\left(\tau_{j}\right)+\left(\tau_{j+1}-\tau_{j}\right) f\left(\tau_{j}, X\left(\tau_{j}\right)\right)+g\left(\tau_{j}, X\left(\tau_{j}\right)\right)\left(W\left(\tau_{j+1}\right)-W\left(\tau_{j}\right)\right)
$$

Define $X\left(\tau_{j+r}\right):=X_{j+r}, \quad r=0,1 ; \delta W_{j}:=\left(W\left(\tau_{j+r}\right)-W\left(\tau_{j+r-1}\right)\right), \quad r=1$.

Equation (10) becomes

$$
X_{j+1}=X_{j}+\delta t f\left(\tau_{j}, X_{j}\right)+g\left(\tau_{j}, X_{j}\right) \delta W_{j}, j=0,1,2, \cdots, L
$$

\subsection{Implementation of the Method}

The method in Equation (11) was considered by [26] using backward difference. In this paper, we shall apply this method to the SDE (3) using discritised interval $[0, T]$ as $0<\tau_{0}<\tau_{1}<\cdots<\tau_{j}<\tau_{j+1}=T$. Let $\delta t=\frac{T}{N}$ be the stepsize defined as $\delta t:=\tau_{j+1}-\tau_{j}$, where $N$ are some integer and $\tau_{j}=j \delta t$. The $\delta t$-space path increment $d W_{j}:=W_{j}-W_{j-1}$ will be approximated by summing the underlying $d t$-space increments as established by [26] using

$$
\text { Winc }=\operatorname{sum}(d W(R *(j-1)+1: R * j))
$$

Wiener increment $d W$ will be generated in MATLAB over the space intervals by using $d W:=\operatorname{sqrt}(d t) * \operatorname{rand}(1, N)$. For computational purpose, we shall assume that $R=1, D t=R * d t$ and $L=N / R$. In this paper, we shall investigate the effect of varying stepsizes in determining the numerical solution of SDE (3) using one-step method of EMM type in Equation (11). This is provided in section 3.0. The absolute errors will be obtained. From this, the mean absolute errors will be determined. Strong order of convergence and the residual for one-step method of EMM type will be determined from mean absolute error by making least square fit using the MALAB commands.

\section{Effect of Varying Stepsizes in Determining Numerical Solution of Stochastic Differential Equations Using One-Step Euler-Maruyama Method}

In this section, we will consider two problems in the form of first order stochastic differential Equation (3) to investigate the effect of varying stepsizes when finding the solution of SDEs using one step method of EMM type in Equation (11). 


\section{Problem 1.}

$$
\mathrm{d} X(t)=\mu X(t) \mathrm{d} t+\sigma X(t) \mathrm{d} W(t), \quad X\left(t_{0}\right)=t_{0}
$$

where $\mu=0.0002$ and $\sigma=0.0001$ are arbitrary values.

The exact solution of the SDE (13) is

$$
X(t)=X_{0} \exp \left(\left(\mu-0.5 \sigma^{2}\right) t+\sigma W(t)\right)
$$

Problem 1 is the Black Scholes option price model with a drift function $f(t, X(t))=\mu X(t)$, drift coefficient $\mu$, diffusion function $g(t, X(t))=\sigma X(t)$ and diffusion coefficient $\sigma$. The model was also used by [26] and [29].

The following stepsizes will be used to carry out our investigation, $2^{-4}, 2^{-5}, 2^{-6}, 2^{-7}, 2^{-8}, 2^{-9}$.

\subsection{Results Showing the Effect of Using Varying Stepsizes When Euler-Maruyama Method Is Applied to Problem 1}

See Figure 1 for the graph of the result on Table 1.

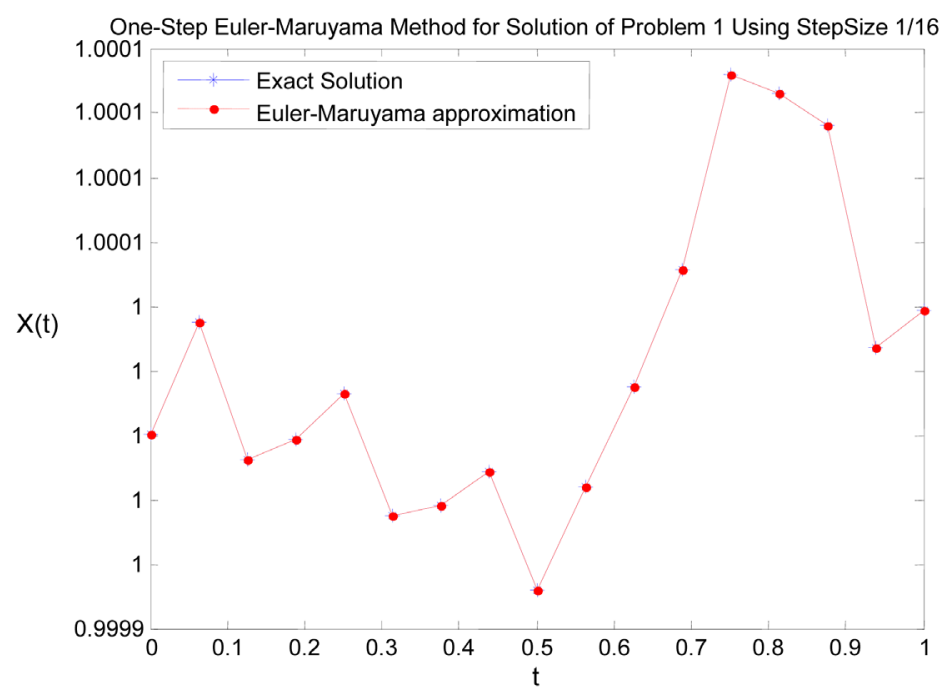

Figure 1. Shows the graph of the result on Table 1.

\begin{tabular}{|c|c|c|c|}
\hline t-value Error & Exact Solution & Numerical Solution & Absolute \\
\hline 0.062500 & 1.000035213412162 & 1.000035213104684 & $3.07477599 \mathrm{e}^{-10}$ \\
\hline 0.125000 & 0.999992194240297 & 0.999992193320025 & $9.20271970 \mathrm{e}^{-10}$ \\
\hline 0.187500 & 0.999998717493128 & 0.999998716864071 & $6.29056918 \mathrm{e}^{-10}$ \\
\hline 0.250000 & 1.000012935644641 & 1.000012935226998 & $4.17643475 e^{-10}$ \\
\hline 0.312500 & 0.999974930990167 & 0.999974930162858 & $8.27309221 \mathrm{e}^{-10}$ \\
\hline 0.375000 & 0.999978328502518 & 0.999978327981927 & $5.20591348 e^{-10}$ \\
\hline 0.437500 & 0.999988796767910 & 0.999988796505013 & $2.62896704 \mathrm{e}^{-}-10$ \\
\hline 0.500000 & 0.999951804297058 & 0.999951803662421 & $6.34636454 \mathrm{e}^{-10}$ \\
\hline 0.562500 & 0.999984007130832 & 0.999984006290135 & $8.40697068 \mathrm{e}^{-10}$ \\
\hline 0.625000 & 1.000014922754158 & 1.000014921748044 & $1.00611364 \mathrm{e}^{-9}$ \\
\hline
\end{tabular}

Table 1. Result of Problem 1 showing the effect of using stepsize $2^{-4}$.

The mean absolute error is 6.366694393911132e-10.

Table 1 shows the exact solution, numerical solution and absolute error for the effect of using stepsize $2^{-4}$. 
See Figure 2 for the graph of the result on Table 2.

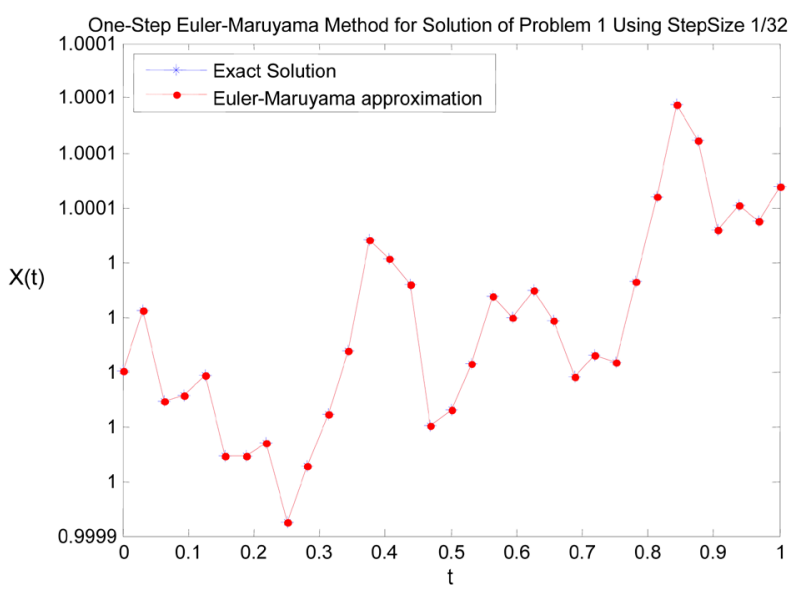

Figure 2. Shows the graph of the result on Table 2.

Table 2. Result of Problem 1 showing the effect of using stepsize $2^{-5}$.

\begin{tabular}{|c|c|c|c|}
\hline t-value Error & Exact Solution & Numerical Solution & Absolute \\
\hline 0.062500 & 0.999989302989965 & 0.999989302508838 & $4.81126805 e^{-10}$ \\
\hline 0.125000 & 0.999998791743439 & 0.999998791544895 & $1.98543737 \mathrm{e}^{-10}$ \\
\hline 0.187500 & 0.999969143626926 & 0.999969143306858 & $3.20068305 e^{-10}$ \\
\hline 0.250000 & 0.999945211010976 & 0.999945210578632 & $4.32343716 e^{-10}$ \\
\hline 0.312500 & 0.999984664319081 & 0.999984663809844 & $5.09236209 e^{-}-10$ \\
\hline 0.375000 & 1.000048162051462 & 1.000048160772574 & $1.27888855 \mathrm{e}^{-9}$ \\
\hline 0.437500 & 1.000031603910325 & 1.000031602873598 & $1.03672670 \mathrm{e}^{-9}$ \\
\hline 0.500000 & 0.999985898306111 & 0.999985896243317 & $2.06279349 e^{-9}$ \\
\hline 0.562500 & 1.000027707908093 & 1.000027705706180 & $2.20191265 e^{-9}$ \\
\hline 0.625000 & 1.000029576187069 & 1.000029574216571 & $1.97049865 e^{-9}$ \\
\hline
\end{tabular}

The mean absolute error is $1.049213882442501 \mathrm{e}-9$.

Table 2 shows the exact solution, numerical solution and absolute error for the effect of using stepsize $2^{-5}$.

See Figure 3 for the graph of the result on Table 3.

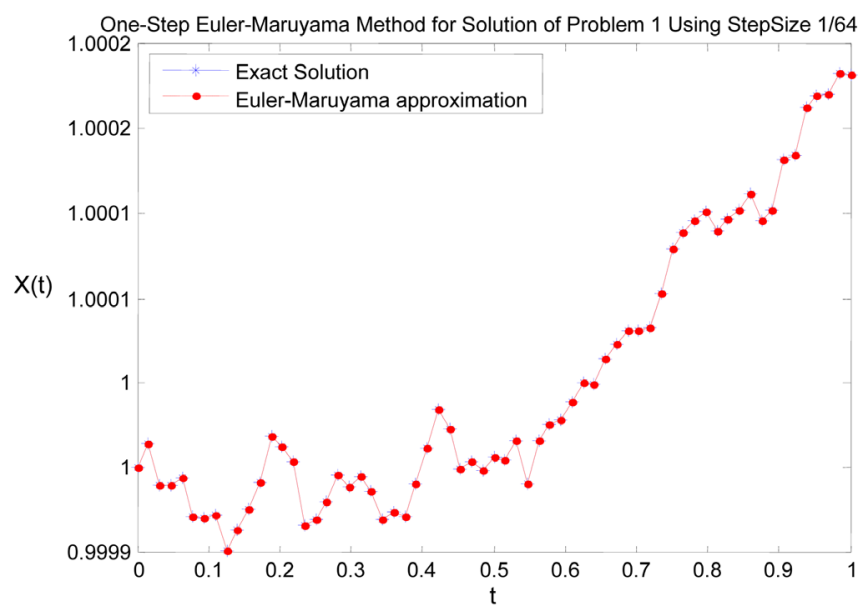

Figure 3. Shows the graph of the result on Table 3. 
Table 3. Result of Problem 1 showing the effect of using stepsize $2^{-6}$.

\begin{tabular}{cccc}
\hline t-value & Exact Solution & Numerical Solution & Absolute Error \\
\hline 0.062500 & 0.999993968111180 & 0.999993968007453 & $1.03727471 \mathrm{e}-10$ \\
0.125000 & 0.999950903398081 & 0.999950903125062 & $2.73019052 \mathrm{e}-10$ \\
0.187500 & 1.000018522444389 & 1.000018521838989 & $6.05399730 \mathrm{e}-10$ \\
0.250000 & 0.999969318836642 & 0.999969317778954 & $1.05768760 \mathrm{e}-9$ \\
0.312500 & 0.999995025525107 & 0.999995024550208 & $9.74898495 \mathrm{e}-10$ \\
0.375000 & 0.999971494315777 & 0.999971493473134 & $8.42642955 \mathrm{e}-10$ \\
0.437500 & 1.000023470014742 & 1.000023468767193 & $1.24754895 \mathrm{e}-9$ \\
0.500000 & 1.000006560271671 & 1.000006558980626 & $1.29104549 \mathrm{e}-9$ \\
0.562500 & 1.000016550151243 & 1.000016548430911 & $1.72033232 \mathrm{e}-9$ \\
0.625000 & 1.000050801848815 & 1.000050800269305 & $1.57950941 \mathrm{e}-9$ \\
\hline
\end{tabular}

The mean absolute error is $9.695811487020478 \mathrm{e}-10$.

Table 3 shows the exact solution, numerical solution and absolute error for the effect of using stepsize $2^{-6}$.

See Figure 4 for the graph of the result on Table 4 .

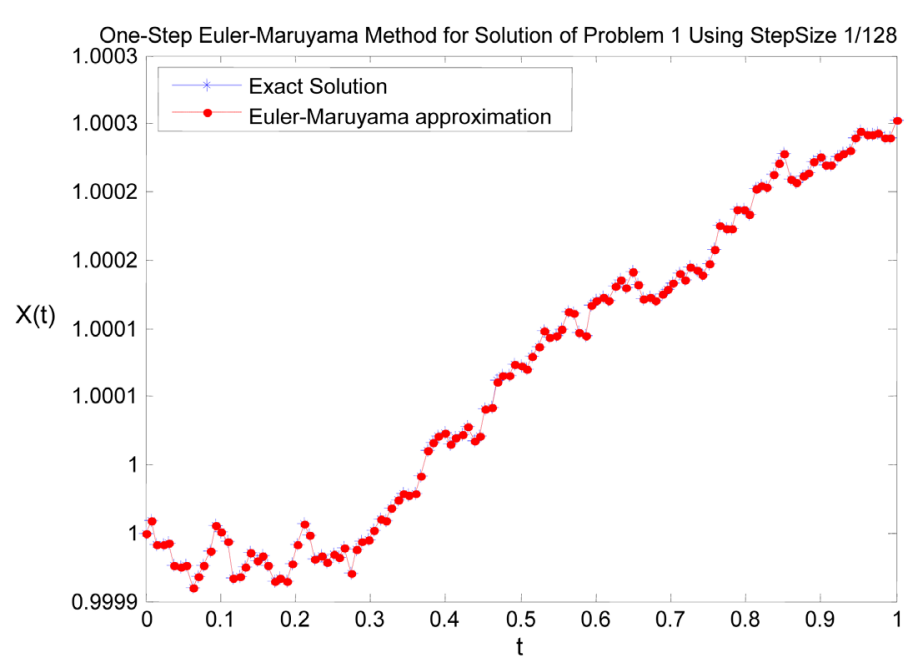

Figure 4. Shows the graph of the result on Table 4.

Table 4. Result of Problem 1 showing the effect of using stepsize $2^{-7}$.

\begin{tabular}{cccc}
\hline t-value & Exact Solution & Numerical Solution & Absolute Error \\
\hline 0.062500 & 0.999960105863295 & 0.999960105702641 & $1.60653491 \mathrm{e}-10$ \\
0.125000 & 0.999967950241945 & 0.999967949695712 & $5.46233170 \mathrm{e}-10$ \\
0.187500 & 0.999964311166688 & 0.999964310727299 & $4.39388304 \mathrm{e}-10$ \\
0.250000 & 0.999983928766161 & 0.999983928116954 & $6.49207244 \mathrm{e}-10$ \\
0.312500 & 1.000010033769263 & 1.000010032994412 & $7.74851072 \mathrm{e}-10$ \\
0.375000 & 1.000060677640722 & 1.000060676842953 & $7.97768518 \mathrm{e}-10$ \\
0.437500 & 1.000067288106194 & 1.000067287456036 & $6.50158150 \mathrm{e}-10$ \\
0.500000 & 1.000122399331946 & 1.000122398556290 & $7.75655762 \mathrm{e}-10$ \\
0.562500 & 1.000162783152388 & 1.000162782441429 & $7.10958847 \mathrm{e}-10$ \\
0.625000 & 1.000180536086851 & 1.000180535284656 & $8.02195865 \mathrm{e}-10$ \\
\hline
\end{tabular}

The mean absolute error is $6.307070421485150 \mathrm{e}-10$.

Table 4 shows the exact solution, numerical solution and absolute error for the effect of using stepsize $2^{-7}$.

See Figure 5 for the graph of the result on Table 5 . 


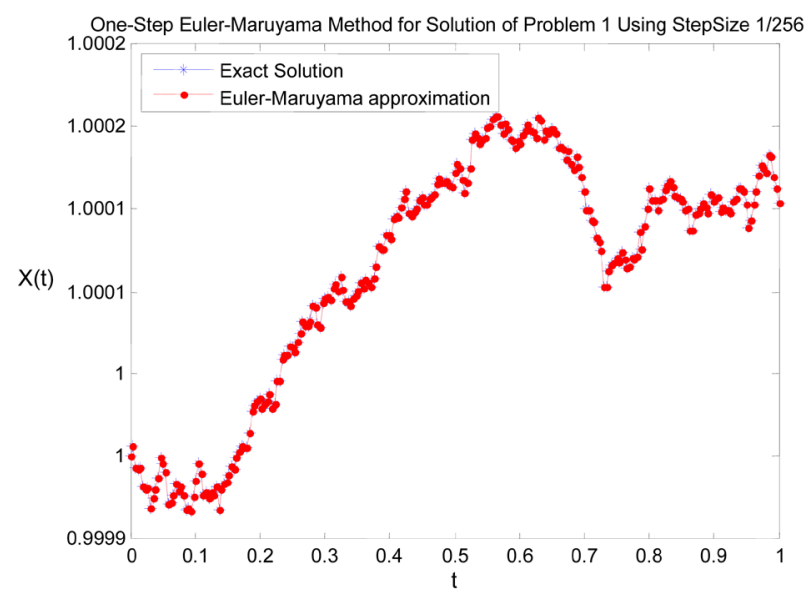

Figure 5. Shows the graph of the result on Table 5.

Table 5. Result of Problem 1 showing the effect of using stepsize $2^{-8}$.

\begin{tabular}{|c|c|c|c|}
\hline t-value & Exact Solution & Numerical Solution & Absolute Error \\
\hline 0.062500 & 0.999972159883026 & 0.999972159601738 & $2.81287882 \mathrm{e}-10$ \\
\hline 0.125000 & 0.999978280985938 & 0.999978280655984 & $3.29953620 \mathrm{e}-10$ \\
\hline 0.187500 & 1.000027372023912 & 1.000027371636413 & $3.87499366 \mathrm{e}-10$ \\
\hline 0.250000 & 1.000065836108020 & 1.000065835744871 & $3.63149066 \mathrm{e}-10$ \\
\hline 0.312500 & 1.000101764246374 & 1.000101763882430 & $3.63944430 e^{-}-10$ \\
\hline 0.375000 & 1.000108726423491 & 1.000108726193473 & $2.30018227 \mathrm{e}^{-10}$ \\
\hline 0.437500 & 1.000148957998176 & 1.000148957704878 & $2.93298275 e^{-10}$ \\
\hline 0.500000 & 1.000172569073211 & 1.000172568980299 & $9.29125665 \mathrm{e}^{-11}$ \\
\hline 0.562500 & 1.000206392121796 & 1.000206391996795 & $1.25001121 \mathrm{e}^{-}-10$ \\
\hline 0.625000 & 1.000193604241676 & 1.000193604296249 & $5.45721246 \mathrm{e}-11$ \\
\hline
\end{tabular}

The mean absolute error is $2.521636677244032 \mathrm{e}-10$.

Table 5 shows the exact solution, numerical solution and absolute error for the effect of using stepsize $2^{-8}$.

See Figure 6 for the graph of the result on Table 6.

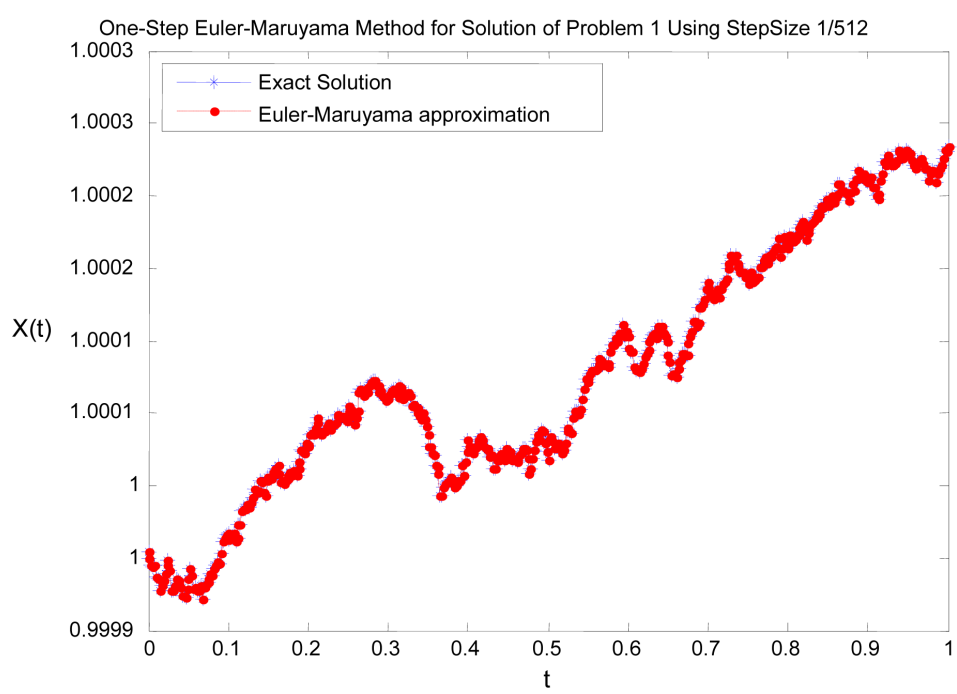

Figure 6. Shows the graph of the result on Table 6. 
Table 6. Result of Problem 1 showing the effect of using stepsize $2^{-9}$.

\begin{tabular}{rrrr}
\hline t-value & Exact Solution & Numerical Solution & Absolute Error \\
\hline 0.062500 & 0.999979464841858 & 0.999979464673978 & $1.67880376 \mathrm{e}-10$ \\
0.125000 & 1.000036197200983 & 1.000036197026108 & $1.74874559 \mathrm{e}-10$ \\
0.187500 & 1.000061346273481 & 1.000061346169660 & $1.03820508 \mathrm{e}-10$ \\
0.250000 & 1.000101309205331 & 1.000101309176948 & $2.83835178 \mathrm{e}-11$ \\
0.312500 & 1.000111004081822 & 1.000111004129163 & $4.73403539 \mathrm{e}-11$ \\
0.375000 & 1.000054041052400 & 1.000054040994347 & $5.80526738 \mathrm{e}-11$ \\
0.437500 & 1.000068042560500 & 1.000068042466253 & $9.42474987 \mathrm{e}-11$ \\
0.500000 & 1.000067650976551 & 1.000067650858876 & $1.17674315 \mathrm{e}-10$ \\
0.562500 & 1.000133899286137 & 1.000133899093955 & $1.92182270 \mathrm{e}-10$ \\
0.625000 & 1.000143970608418 & 1.000143970444947 & $1.63471015 \mathrm{e}-10$ \\
\hline
\end{tabular}

The mean absolute error is $1.147927086719847 \mathrm{e}-10$.

Table 6 shows the exact solution, numerical solution and absolute error for the effect of using stepsize $2^{-9}$.

\section{Problem 2.}

$$
\mathrm{d} X(t)=-\left(a+b^{2} X(t)\right)\left(\left(1-X^{2}(t)\right) \mathrm{d} t+b\left(1-X^{2}(t)\right)\right) \mathrm{d} W(t)
$$

where $a=0.0002, b=0.0001, t \in[0,1]$ and $X_{0}=-2$ are arbitrary values. They are used for better accuracy.

The true solution is

$$
X(t)=\frac{\left(1+X_{0}\right) \mathrm{e}^{(-2 a t+2 b W(t))}+X_{0}-1}{\left(\left(1+X_{0}\right) \mathrm{e}^{(-2 a t+2 b W(t))}-X_{0}+1\right)}
$$

Problem 2 was used by [25] with constants $a=1, b=2$ and $X_{0}=0$.

\subsection{Results Showing the Effect of Using Varying Stepsizes When Euler-Maruyama Method Is Applied to Problem 2}

See Figure 7 for the graph of the result on Table 7.

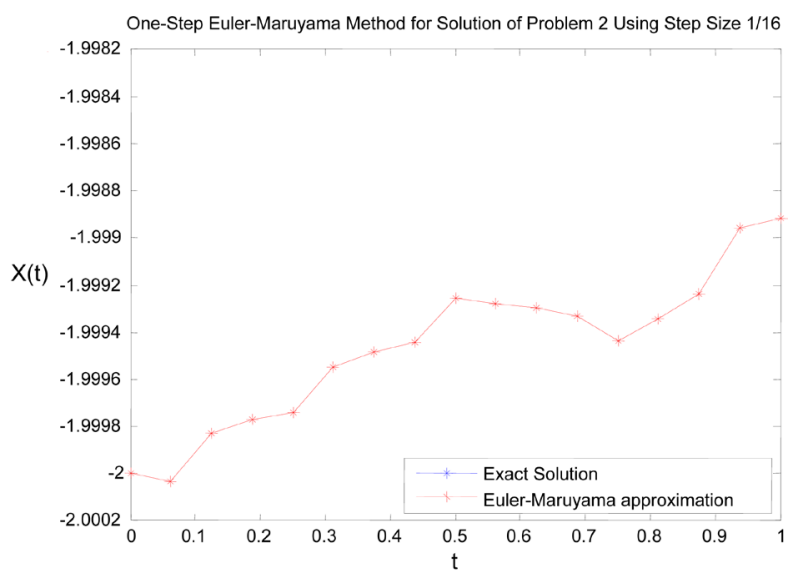

Figure 7. Shows the graph of the result on Table 7. 
Table 7. Result of Problem 2 showing the effect of using stepsize $2^{-4}$.

\begin{tabular}{cccc}
\hline t-value & Exact Solution & Numerical Solution & Absolute Error \\
\hline 0.062500 & -2.000030639939910 & -2.000030643064053 & $3.12414317 \mathrm{e}-9$ \\
0.125000 & -1.999826604550993 & -1.999826583667489 & $2.08835040 \mathrm{e}-8$ \\
0.187500 & -1.999771190197802 & -1.999771171017104 & $1.91806975 \mathrm{e}-8$ \\
0.250000 & -1.999738855905664 & -1.999738839777066 & $1.61285976 \mathrm{e}-8$ \\
0.312500 & -1.999549931800135 & -1.9995498956620500 & $3.61796346 \mathrm{e}-8$ \\
0.375000 & -1.999485167195670 & -1.999485131968585 & $3.52270857 \mathrm{e}-8$ \\
0.437500 & -1.999441604632085 & -1.999441571887793 & $3.27442917 \mathrm{e}-8$ \\
0.500000 & -1.999255786341376 & -1.999255734318596 & $5.20227794 \mathrm{e}-8$ \\
0.562500 & -1.999277377750062 & -1.999277329159688 & $4.85903735 \mathrm{e}-8$ \\
0.625000 & -1.999295108719981 & -1.999295063663776 & $4.50562045 \mathrm{e}-8$ \\
\hline
\end{tabular}

The mean absolute error is $3.091373117491969 \mathrm{e}-8$.

Table 7 shows the exact solution, numerical solution and absolute error for the effect of using stepsize $2^{-4}$.

See Figure 8 for the graph of the result on Table 8.

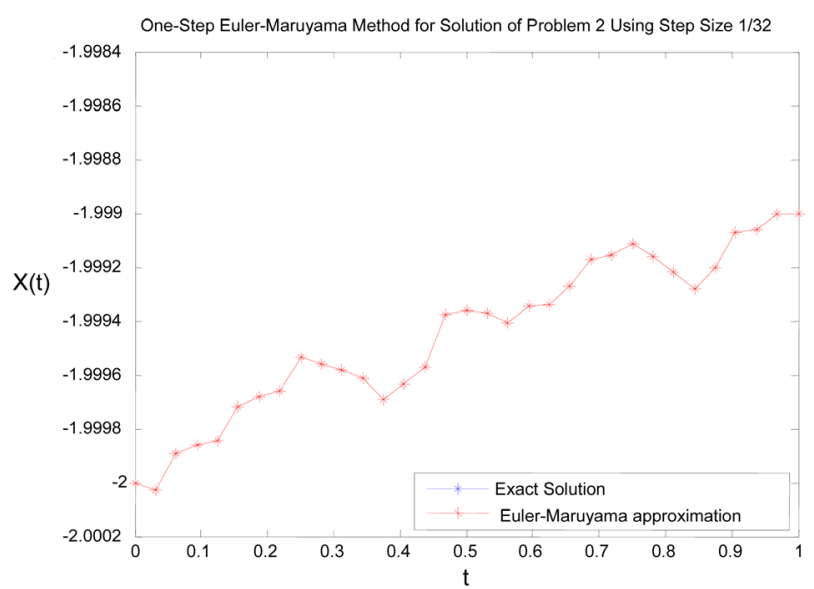

Figure 8. Shows the graph of the result on Table 8.

Table 8. Result of Problem 2 showing the effect of using stepsize $2^{-5}$.

\begin{tabular}{cccc}
\hline t-value & Exact Solution & Numerical Solution & Absolute Error \\
\hline 0.062500 & -1.999892917380804 & -1.999892908128028 & $9.25277566 \mathrm{e}-9$ \\
0.125000 & -1.999846392834979 & -1.999846386521514 & $6.31346508 \mathrm{e}-9$ \\
0.187500 & -1.999682499484875 & -1.999682485395495 & $1.40893797 \mathrm{e}-8$ \\
0.250000 & -1.999535775997482 & -1.999535755103327 & $2.08941555 \mathrm{e}-8$ \\
0.312500 & -1.999579115423757 & -1.999579097646508 & $1.77772492 \mathrm{e}-8$ \\
0.375000 & -1.999694550513440 & -1.999694531148642 & $1.93647987 \mathrm{e}-8$ \\
0.437500 & -1.999569940134597 & -1.999569919321205 & $2.08133912 \mathrm{e}-8$ \\
0.500000 & -1.999357977043176 & -1.999357935211449 & $4.18317270 \mathrm{e}-8$ \\
0.562500 & -1.999408364465035 & -1.999408325355391 & $3.91096437 \mathrm{e}-8$ \\
0.625000 & -1.999339028019188 & -1.999338990093991 & $3.79251974 \mathrm{e}-8$ \\
\hline
\end{tabular}

The mean absolute error is $2.273717831791089 \mathrm{e}-8$.

Table 8 shows the exact solution, numerical solution and absolute error for the effect of using stepsize $2^{-5}$. 
See Figure 9 for the graph of the result on Table 9 .

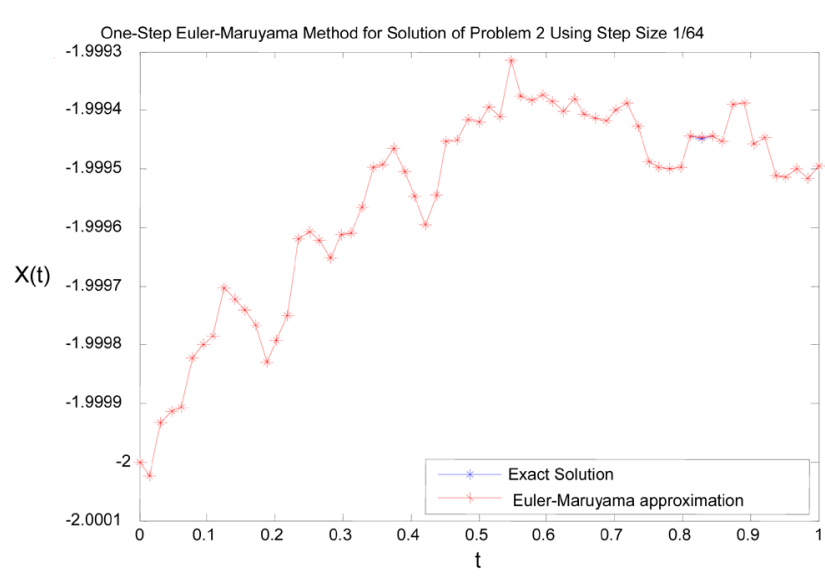

Figure 9. Shows the graph of the result on Table 9.

Table 9. Result of Problem 2 showing the effect of using stepsize $2^{-6}$.

\begin{tabular}{ccccc}
\hline t-value & Exact Solution & Numerical Solution & Absolute Error \\
\hline 0.062500 & -1.999906910993895 & -1.999906908359276 & $2.63461808 \mathrm{e}-9$ \\
0.125000 & -1.999702767364218 & -1.999702758533899 & $8.83031892 \mathrm{e}-9$ \\
0.187500 & -1.999830588766838 & -1.999830580080542 & $8.68629546 \mathrm{e}-9$ \\
0.250000 & -1.999608061287530 & -1.999608042553839 & $1.87336919 \mathrm{e}-8$ \\
0.312500 & -1.999610182559322 & -1.999610165808479 & $1.67508436 \mathrm{e}-8$ \\
0.375000 & -1.999464678473431 & -1.999464660611312 & $1.78621189 \mathrm{e}-8$ \\
0.437500 & -1.999545553506756 & -1.999545533746613 & & \\
0.500000 & -1.999419912678686 & -1.999419890196399 & & $2.24822863 \mathrm{e}-8$ \\
0.562500 & -1.999374919082127 & -1.999374891267540 & & $2.78145871 \mathrm{e}-8$ \\
0.625000 & -1.999402649038457 & -1.999402624540933 & \\
\hline
\end{tabular}

The mean absolute error is $1.680524275293749 \mathrm{e}-8$.

Table 9 shows the exact solution, numerical solution and absolute error for the effect of using stepsize $2^{-6}$.

See Figure 10 for the graph of the result on Table 10.

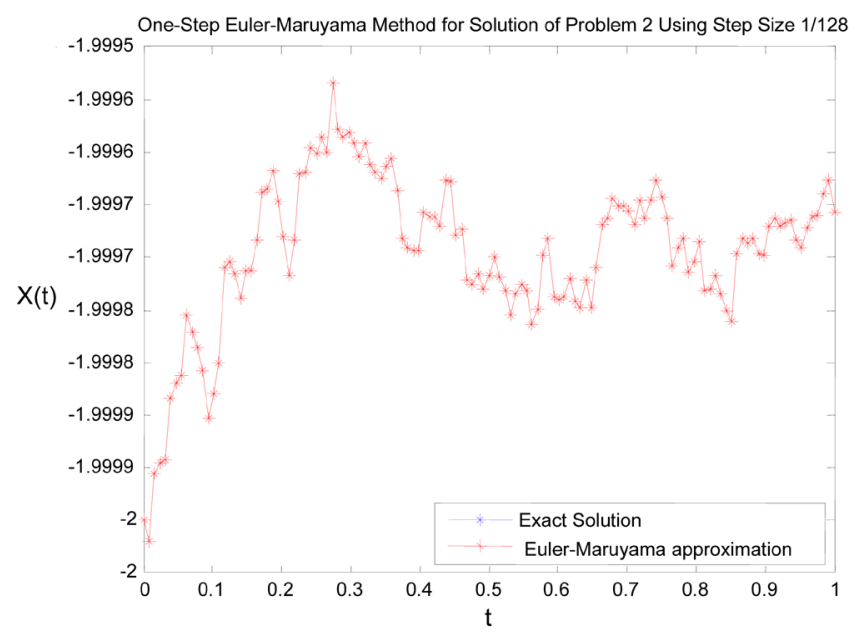

Figure 10. Shows the graph of the result on Table 10. 
Table 10. Result of Problem 2 showing the effect of using stepsize $2^{-7}$.

\begin{tabular}{cccc}
\hline t-value & Exact Solution & Numerical Solution & Absolute Error \\
\hline 0.062500 & -1.999805341404871 & -1.999805337513111 & $3.89176003 \mathrm{e}-9$ \\
0.125000 & -1.999753891446814 & -1.999753882755321 & $8.69149241 \mathrm{e}-9$ \\
0.187500 & -1.999668007898771 & -1.999667999884824 & $8.01394706 \mathrm{e}-9$ \\
0.250000 & -1.999651870477482 & -1.999651860214105 & $1.02633777 \mathrm{e}-8$ \\
0.312500 & -1.999655185128882 & -1.999655173868698 & $1.12601839 \mathrm{e}-8$ \\
0.375000 & -1.999732080887753 & -1.999732070781591 & $1.01061621 \mathrm{e}-8$ \\
0.437500 & -1.999676933687320 & -1.999676925131407 & $8.55591287 \mathrm{e}-9$ \\
0.500000 & -1.999767219155966 & -1.999767210691419 & $8.46454751 \mathrm{e}-9$ \\
0.562500 & -1.999813341382202 & -1.999813334738500 & $6.64370181 \mathrm{e}-9$ \\
0.625000 & -1.999791597710221 & -1.999791590169479 & $7.54074203 \mathrm{e}-9$ \\
\hline
\end{tabular}

The mean absolute error is $8.343182744674494 \mathrm{e}-9$.

Table 10 shows the exact solution, numerical solution and absolute error for the effect of using stepsize $2^{-7}$.

See Figure 11 for the graph of the result on Table 11.

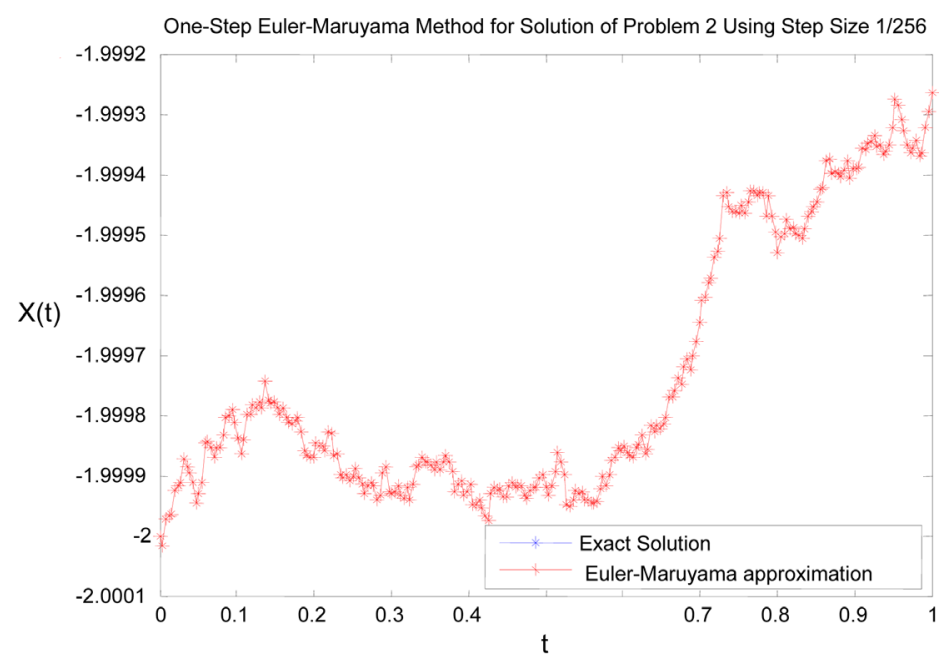

Figure 11. Shows the graph of the result on Table 11.

Table 11. Result of Problem 2 showing the effect of using stepsize $2^{-8}$.

\begin{tabular}{|c|c|c|c|}
\hline t-value & Exact Solution & Numerical Solution & Absolute Error \\
\hline 0.062500 & -1.999841496174841 & -1.999841492043785 & $4.13105594 \mathrm{e}^{-9}$ \\
\hline 0.125000 & -1.999784874982539 & -1.999784870148436 & $4.83410290 \mathrm{e}^{-9}$ \\
\hline 0.187500 & -1.999857131369450 & -1.999857126530599 & $4.83885132 \mathrm{e}^{-9}$ \\
\hline 0.250000 & -1.999897512575721 & -1.999897508515622 & $4.06009937 \mathrm{e}^{-9}$ \\
\hline 0.312500 & -1.999930285134005 & -1.999930281503511 & $3.63049413 e^{-9}$ \\
\hline 0.375000 & -1.999876177386879 & -1.999876175259421 & $2.12745799 \mathrm{e}-9$ \\
\hline 0.437500 & -1.999921851349309 & -1.999921848982462 & $2.36684672 \mathrm{e}^{-9}$ \\
\hline 0.500000 & -1.999917674573231 & -1.999917674818573 & $2.45341969 \mathrm{e}-10$ \\
\hline 0.562500 & -1.999944122996693 & -1.999944123256830 & $2.60136801 \mathrm{e}^{-10}$ \\
\hline 0.625000 & -1.999830784974864 & -1.999830786914828 & $1.93996397 \mathrm{e}-9$ \\
\hline
\end{tabular}

The mean absolute error is $2.843435109589621 \mathrm{e}-9$.

Table 11 shows the exact solution, numerical solution and absolute error for the effect of using stepsize $2^{-7}$. 
See Figure 12 for the graph of the result on Table 12.

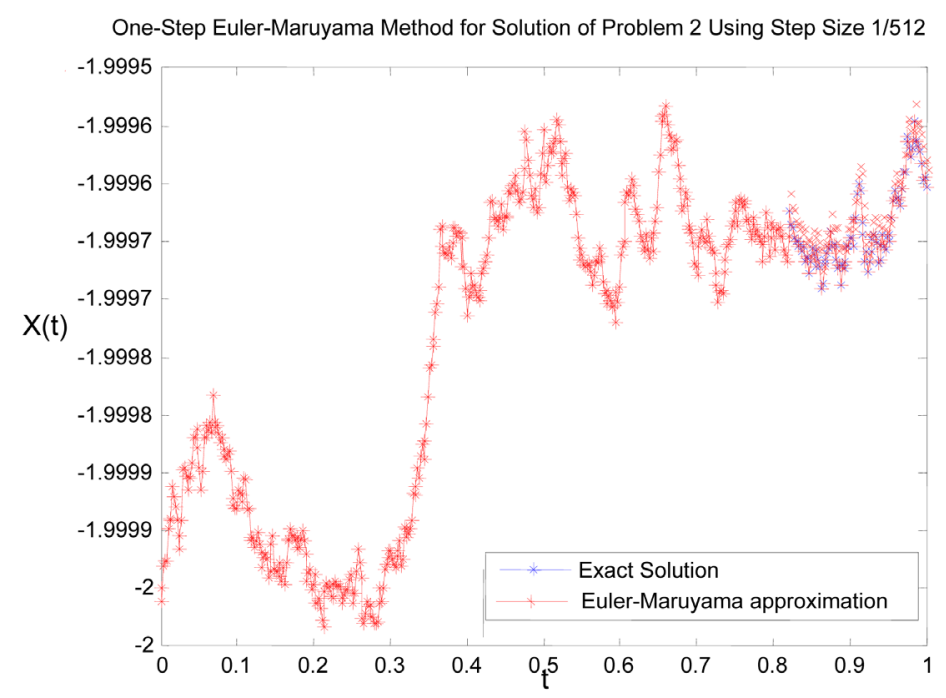

Figure 12. shows the graph of the result on Table 12.

Table 12. Result of problem 2 showing the effect of using stepsize $2^{-9}$.

\begin{tabular}{|c|c|c|c|}
\hline t-value & Exact Solution & Numerical Solution & Absolute Error \\
\hline 0.062500 & -1.999863407270136 & -1.999863404946251 & $2.32388553 e^{-9}$ \\
\hline 0.125000 & -1.999958592655718 & -1.999958590662368 & $1.99335082 \mathrm{e}-9$ \\
\hline 0.187500 & -1.999959037106798 & -1.999959036084589 & $1.02220876 \mathrm{e}^{-9}$ \\
\hline 0.250000 & -2.000003915981924 & -2.000003916122282 & $1.40357947 e^{-10}$ \\
\hline 0.312500 & -1.999957999627532 & -1.999958000650131 & $1.02259934 \mathrm{e}^{-9}$ \\
\hline 0.375000 & -1.999712179640226 & -1.999712178747598 & $8.92628638 e^{-10}$ \\
\hline 0.437500 & -1.999679195925699 & -1.999679194613133 & $1.31256606 \mathrm{e}^{-9}$ \\
\hline 0.500000 & -1.999603058636733 & -1.999603056922618 & $1.71411507 e^{-9}$ \\
\hline 0.562500 & -1.999726729199231 & -1.999726727095061 & $2.10416973 e^{-9}$ \\
\hline 0.625000 & -1.999681957561421 & -1.999681955778895 & $1.78252613 e^{-9}$ \\
\hline
\end{tabular}

The mean absolute error is $1.430840801397437 \mathrm{e}-9$.

Table 12 shows the exact solution, numerical solution and absolute error for the effect of using stepsize $2^{-9}$.

\section{Determination of Strong Order of Convergence of the Method}

In this section, it will be wise to determine the strong order of convergence (SOC) and the residual of the method as this property is important to examine how good or how accurate our approximations are. SOC is a property of solution that is indispensable in examining the accuracy of any numerical method for solution of SDEs.

The issues of convergence and SOC of SDEs have also been examined by [20] [23] [25]-[27] [29] [31] for one step Method. The papers and book gives the theoretical background of SDEs and SOC. [27] examined the SOC for one step method of Milstein (MLSTM) type. The vital tool in the determination of SOC is the mean absolute error (MAE) or strong error. MAE is the mean of the absolute value of difference between the exact solution and numerical solution of a given stochastic differential equation. It serves as a tool for assessing the effect of varying step sizes. MAE will be determined for one-step method of EMM type just developed using varying stepsizes. The SOC of the method will be obtained using the MAE. 
Table 13. Results of the MAE of one step method of EMM type for Problem 1.

\begin{tabular}{ccc}
\hline & Stepsize & Problem 1 \\
\cline { 2 - 2 } $2^{-4}$ & MAE of One Step Method EMM Type \\
$2^{-5}$ & $6.36669439 \mathrm{e}-10$ \\
$2^{-6}$ & $1.04921388 \mathrm{e}-9$ \\
$2^{-7}$ & $9.69581149 \mathrm{e}-10$ \\
$2^{-8}$ & $6.30707042 \mathrm{e}-10$ \\
$2^{-9}$ & $2.52163668 \mathrm{e}-10$ \\
& $1.14792709 \mathrm{e}-10$ \\
\hline
\end{tabular}

Table 13 shows the results of mean absolute error of one-step method of EMM type applied to Problem 1 with varying stepsizes.

Table 14. Results of the MAE of one-step method of EMM type for Problem 2.

\begin{tabular}{cc}
\hline Stepsize & Problem 2 \\
\cline { 2 - 2 } & MAE of One Step Method EMM Type \\
\hline $2^{-4}$ & $3.09137312 \mathrm{e}-8$ \\
$2^{-5}$ & $2.27371783 \mathrm{e}-8$ \\
$2^{-6}$ & $1.68052428 \mathrm{e}-8$ \\
$2^{-7}$ & $8.34318274 \mathrm{e}^{-9}$ \\
$2^{-8}$ & $2.84343511 \mathrm{e}-9$ \\
$2^{-9}$ & $1.43084080 \mathrm{e}-9$ \\
\hline
\end{tabular}

Table 14 shows the results of mean absolute error of one-step method of EMM type applied to Problem 2 with varying stepsizes.

Table 15. Results of the strong order of convergence of one-step method of Euler-Maruyama type for Problems $\mathbf{1}$ and $\mathbf{2}$.

\begin{tabular}{ccc}
\hline & One-step Method of EMM Type & \\
\hline & Problem 1 & Problem 2 \\
\hline SOC & 0.5471 & 0.9193 \\
RESIDUAL & 1.0975 & 0.6067 \\
\hline
\end{tabular}

Table 15 shows the strong order of convergence of one-step method of EMM type for Problems 1 and 2.

\section{Discussion}

In this paper effort has been made to discuss the derivation of one-step method of Euler-Maruyama type. This method was applied to two problems in the form of first order SDEs. The method was used to determine the numerical solution of the two problems. Absolute errors were calculated using the numerical approximation and the corresponding exact solution. Mean absolute errors were also determined. To determine the accuracy of the method, strong order of convergence and residuals were obtained for each problem.

\section{Conclusion}

In this paper, two problems in the form of first order SDEs have been considered. One-step method of EulerMaruyama type for solution of general first order SDEs has been derived. The absolute errors between the exact solution and numerical solution can be observed. The mean absolute error for varying stepsizes has been deter- 
mined. The result shows that the mean absolute error generally decreases as the stepsizes decreases. The accuracy of the method is determined by finding the strong order of convergence of the method. The result shows that the strong order of convergence is 0.5471 and the residual is 1.0975 for Problem 1 while the strong order of convergence is 0.9193 and the residual is 0.6067 for Problem 2. The effect of the varying stepsizes can also be seen by observing the behaviour of the exact solution and numerical solution using graphical method as indicated in Figures 1-12. The results are obtained using MATLAB as supporting tool.

\section{References}

[1] Rezaeyan, R. and Farnoosh, R. (2010) Stochastic Differential Equations and Application of Kalman-Bucy Filter in Modeling of RC Circuit. Applied Mathematical Sciences, Stochastic Differential Equations, 4, 1119-1127.

[2] Awoyemi, D.O. (1999) A Class of Continuous Linear Methods for General Second Order Initial Value Problems In Ordinary Differential Equations. International Journal of Computer Mathematics, 72, 29-37. http://dx.doi.org/10.1080/00207169908804832

[3] Awoyemi, D.O. (2001) A New Sixth-Order Algorithms for General Second Order Ordinary Differential Equations. International Journal of Computer Mathematics, 77, 177-124. http://dx.doi.org/10.1080/00207160108805054

[4] Awoyemi, D.O. (2003) A P-Stable Linear Multistep Method for Solving Third Order Ordinary Differential Equations. International Journal of Computer Mathematics, 80, 85-991. http://dx.doi.org/10.1080/0020716031000079572

[5] Awoyemi, D.O. (2005) An Algorithm Collocation Methods for Direct Solution of Special and General Fouth Order Initial Value Problems of Ordinary Differential Equations. Journal of the Nigerian Association of Mathematical Physics, 6, 271-238.

[6] Awoyemi, D.O., Kayode, S.J. and Adoghe, L.O. (2013) A Four-Point Fully Implicit Method for the Numerical Integration of Third-Order Ordinary Differential Equations. International Journal of Physical Sciences, 9, 7-12. http://dx.doi.org/10.5897/IJPS2013.4019

[7] Kayode, S.J. (2005) An Improved Numerov Method for Direct Solution of General Second Order Initial Value Problems of Ordinary Differential Equations. Proc. Semiar, Nat. Math. Centre, Abuja.

[8] Kayode, S.J. (2009) A Zero Stable Method for Direct Solution of Fourth Order Ordinary Differential Equations. American Journal of Applied Sciences, 5, 1461-1466.

[9] Kayode, S.J. (2011) A Class of One-Point Zero-Stable Continuous Hybrid Methods for Direct Solution of Second Order Differential Equations. African Journal of Mathathematics and Computer Science Researche, 4, 93-99.

[10] Kayode, S.J. and Adeyeye, O. (2011) A 3-Step Hybrid Method for Direct Solution of Second Order Initial Value Problems. Australian Journal of basic and Applied Sciences, 5, 2121-2126.

[11] Kayode, S.J. and Adeyeye, O. (2013) Two-Step Two-Point Hybrid Methods for General Second Order Differential Equations. African Journal of Mathematics and Computer Science Research, 6, 191-196.

[12] Kayode, S.J. and Obarhua, F.O. (2013) Continuous Y-Function Hybrid Methods for Direct Solution of Differential Equations. International Journal of Differential Equations and Applications, 12, 37-48.

[13] Kayode, S.J. (2014) Symmetric Implicit Multiderivative Numerical Integrators for Direct Solution of Fifth-Order Differential Equations. Thammasat International Journal of Science and Technology, 19, 1-8.

[14] Kayode, S.J., Duromola, M.K. and Bolaji, B. (2014) Direct Solution of Initial Value Problems of Fourth Order Ordinary Differential Equations Using Modified Implicit Hybrid Block Method. Journal of Scientific Research and Reports, 3, 2790-2798. http://dx.doi.org/10.9734/jsrr/2014/11953

[15] Olabode, B.T. (2007) Some Linear Multistep Methods for Special General Third Order Initial Value Problems of Ordinary Differential Equations. PhD Thesis, Federal University of Technology, Akure. (Unpublished)

[16] Olabode, B.T. (2013) A Block Predictor-Corrector Method for the Direct Solution of General Fifth Order Ordinary Differential Equations. Canadian Journal on Computing in Mathematics, Natural Science, Engineering and Medicine, 4, 133-139.

[17] Olabode, B.T. (2013) Block Multistep Method for Direct Solution of Third Order Ordinary Differential Equations. FUTA Journal of Research in Science, 2, 194-200.

[18] Onumanyi, P., Sirisena, U.W. and Dauda, Y. (2001) Towards Uniformly Accurate Continuous Finite Difference Approximation of Ordinary Differential Equations. Bagale Journal of Pure and Applied Sciences, 1, 5-8.

[19] Yahaya, Y.A. and Badmus, A.M. (2009) A Class of Collocation Methods for General Second Order Ordinary Differential Equations. African Journal of Mathematics and Computer Science Research, 2, 69-72.

[20] Akinbo, B.J., Faniran, T. and Ayoola, E.O. (2015) Numerical Solution of Stochastic Differential Equations. International Journal of Advanced Research in Science, Engineering and Technology, 2, 608-616. 
[21] Anna, N. (2010) Economical Runge-Kutta Methods with Week Second Order for Stochastic Differential Equations. International Journal of Contemporary Mathematical Sciences, 5, 1151-1160.

[22] Bokor, R.H. (2003) Stochastically Stable One Step Approximations of Solutions of Stochastic Ordinary Differential Equations. Journal of Applied Numerical Mathematics, 44, 21-39. http://dx.doi.org/10.1016/S0168-9274(02)00141-1

[23] Burrage, K. (2004) Numerical Methods for Strong Solutions of Stochastic Differential Equations: An Overview. Proceedings of the Mathematical Physical and Engineering Science, 460, 373-402.

[24] Burrage, K., Burrage, P. and Mitsui, T. (2000) Numerical Solutions of Stochastic Differential Equations-Implementation and Stability Issues. Journal of Computational \& Applied Mathematics, 125, 171-182. http://dx.doi.org/10.1016/S0377-0427(00)00467-2

[25] Fadugba, S.E., Adegboyegun, B.J. and Ogunbiyi, O.T. (2013) On Convergence of Euler Maruyama and Milstein Scheme for Solution of Stochastic Differential Equations. International Journal of Applied Mathematics and Modeling, 1, 9-15.

[26] Higham, D.J. (2001) An Algorithmic Introduction to Numerical Simulation of Stochastic Differential Equations. SIAM Review, 43, 525-546. http://dx.doi.org/10.1137/S0036144500378302

[27] Kayode, S.J. and Ganiyu, A.A. (2015) Effect of Varying Stepsize in Numerical Approximation of Stochastic Differential Equations Using One Step Milstein Method. Applied and Computational Mathematics, 4, 351-362. http://dx.doi.org/10.11648/j.acm.20150405.14

[28] Platen, E. (1992) An Introduction to Numerical Methods of Stochastic Differential Equations. Acta Numerica, 8, 197246. http://dx.doi.org/10.1017/S0962492900002920

[29] Sauer, T. (2013) Computational Solution of Stochastic Differential Equations. Wiley Interdisciplinary Reviews: Computational Statistics, 5, 362-371. http://dx.doi.org/10.1002/wics.1272

[30] Saito, Y. and Mitsui, T. (1996) Stability Analysis of Numerical Schemes for Stochastic Differential Equations. SIAM Journal on Numerical Analysis, 33, 2254-2267. http://dx.doi.org/10.1137/s0036142992228409

[31] Lactus, M.L. (2008) Simulation and Inference for Stochastic Differential Equations with R Examples. Springer Science + Buisiness Media, LLC, New York, 61-62. 